\title{
Advantages and limitations of current premature ejaculation assessment and diagnostic methods: a review
}

\author{
Shanzun Wei ${ }^{1,2}$, Changjing $\mathrm{Wu}^{1}$, Botao Yu${ }^{1,2}$, Ming $\mathrm{Ma}^{1,2}$, Feng Qin ${ }^{1}$, Jiuhong Yuan ${ }^{1,2}$ \\ ${ }^{1}$ The Andrology Laboratory, ${ }^{2}$ Department of Urology, West China Hospital, Sichuan University, Chengdu 610041, China \\ Contributions: (I) Conception and design: S Wei, C Wu , B Yu; (II) Administrative support: F Qin, J Yuan; (III) Provision of study material or patients: \\ S Wei, C Wu; (IV) Collection and assembly of data: S Wei, C Wu, B Yu; (V) Data analysis and interpretation: S Wei, C Wu, M Ma; (VI) Manuscript \\ writing: All authors; (VII) Final approval of manuscript: All authors. \\ Correspondence to: Dr./Prof. Jiuhong Yuan. The Andrology Laboratory, Department of Urology, West China Hospital, Sichuan University, Chengdu \\ 610041, China. Email: jiuhongyuan2107@163.com.
}

\begin{abstract}
Premature ejaculation (PE) is the most common male sexual dysfunction worldwide. Characteristic symptoms of $\mathrm{PE}$ are unexpected, rapid, complete ejaculation, which negatively impacts the sexual act for both sexual partners. Despite the existence of a definitive PE classification system and various diagnostic tools, diagnosing $\mathrm{PE}$ is still challenging due to the limitations associated with the assessment of this condition. Hence, it is necessary to review the diagnostic methods and processes of the physical examination that are currently performed in the medical setting. It is also important to analyze any controversial results of each main $\mathrm{PE}$ assessment method and propose novel diagnostic and assessment methods. To date, it is important to verify the accuracy of the PE evaluation method due to the ambiguity of previous definitions and proven invalidity of current examining techniques. Clinical diagnosis is based mainly on the patient history, patient-reported outcome scores, and diagnostic tools. Introduction of intravaginal ejaculatory latency time, penile biothesiometry, and the electrophysiological test provided objective means of evaluating PE. Due to the controversial and inconclusive findings in PE psychogenic and neurogenic etiology, utilizing a single parameter to describe and qualify PE using the aforementioned diagnostic methods provides valuable, but insufficient information for PE diagnosis. There is still a lack of a feasible and plausible means of objective measurement to evaluate the ejaculatory latency and control over ejaculation. Consequently, a comprehensive penile stimulation that simulates sexual intercourse could be useful to record intensity and duration parameters before the ejaculatory threshold, providing a more accurate method of describing and diagnosing PE versus a single chronological observation.
\end{abstract}

Keywords: Premature ejaculation (PE); intravaginal estimated latency time (IELT); patient-report outcome questionnaire; penile sensitivity

Submitted Jul 03, 2019. Accepted for publication Nov 29, 2019.

doi: 10.21037/tau.2019.12.08

View this article at: http://dx.doi.org/10.21037/tau.2019.12.08

\section{Introduction}

Premature ejaculation (PE) is one of the most common male sexual dysfunction worldwide (1). Its estimated prevalence rate is $20-40 \%$ in the Asia-Pacific and continental regions $(1,2)$. In some areas of China, the latest survey revealed that prevalence rates range from $10.98 \%$ to $21.39 \%$, and the total complaint rate was $34.62 \%$ (3). The characteristic symptoms of PE are unexpected, rapid, and inability to delay ejaculation. It also causes persistent psychological stress to both sexual partners and negatively impacts their mental state and sexual experiences.

The initially classification of $\mathrm{PE}$ was as primary (lifelong) or secondary (acquired), and this classification was expanded by McMahon et al. to include lifelong PE (LPE), acquired $\mathrm{PE}$ (APE), variable PE (VPE), and subjective PE (SPE) (4). This classification system describes the particular characteristics of each PE category, but it was believed that 
Table 1 Features of diagnose and evaluating methods for premature ejaculation

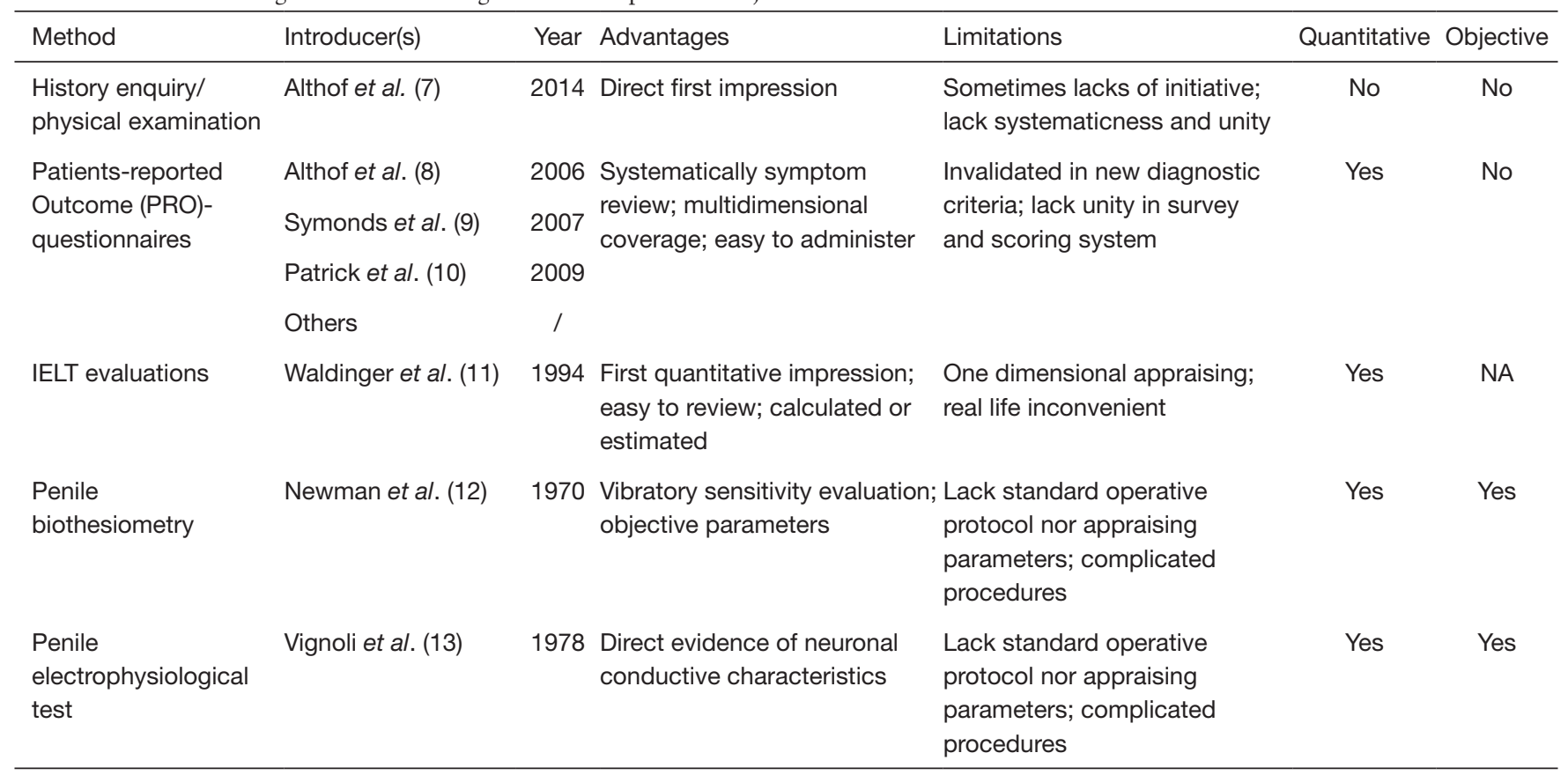

NA, not available.

this system failed in the elucidation of this complicated condition (5). Additionally, despite different domains of distress, such as sexual distress, interpersonal difficulties, inability to delay or withhold ejaculatory impulses as described in this classification system, current PE type and diagnosis were increasingly relying solely on the calculation of the duration from vaginal penetration to ejaculation or the intravaginal estimated latency time (IELT) (4). This trend makes the IELT the most important parameter for $\mathrm{PE}$ determination and diagnosis.

PE was also believed to be a psychological problem (5). Nevertheless, the classification of PE patients was firstly based on doctors' impressions and their evaluation. The initial assessment of patients includes a medical history, physical examination, questionnaires, and other diagnostic tools. Overall, these assessment methods were less subjective (6). Thus, it would be essential to develop a quantitative and systematic measuring scheme to establish an objective approach for the diagnosis of PE, which could be used in the treatment of this condition.

To date, several measures have been applied for the evaluation of PE. These methods include history enquiry/ physical examination (7), patients-reported outcome (PRO)-questionnaires (8-10), IELT evaluations (11), penile biothesiometry (12), and penile electrophysiological test (13). The features, advantages, and limitations of these methods are listed in Table 1. Based on a comprehensive review of the literature, our study summarizes the main methods in order to elucidate the advantages and disadvantages associated with each.

\section{History inquiry}

A patient history inquiry regarding issues with ejaculation can be a difficult endeavour. The act of avoidance or an unwillingness to discuss sexual issues in clinical practice, either by patient or consultant, may aggravate any preexisting misunderstanding over ejaculatory-related issues $(1,14)$. In a survey of 12,113 men diagnosed with PE, Porst $e t$ al. demonstrated that the concomitance of $\mathrm{PE}$ with psychological disturbances is $23 \%$, which is higher than it is in individuals without PE in the United States, Germany, and Italy (14). Regarding the outpatient PE visiting rate, Lee et al. reported that most men are reluctant to seek professional advice (15). He suggests that this is probably due to historical culture, reasons related to social psychology, and ignorance of the prevalence and diagnostic criteria of this condition (15). Similar social psychology, historical, and cultural effects are more evident in Asia, which may explain why the self-reported rate of ejaculatory abnormality is only $2 \%$ in these regions, while in Europe 
and the United States, it can reach $81.9 \%(14,16)$.

Furthermore, Shechter et al. pointed out that factors such as clinical practice, educational background, and the own sexual experiences of the physicians may constitute reasons for an underlying biased diagnosis of PE (17). These findings suggest that the misconceptions regarding the subjective ejaculatory symptoms and clinical history may lead to a biased patient history regarding $\mathrm{PE}$ and erroneous diagnosis in clinical practice. In these cases, the patient history must be rectified before reaching a diagnosis.

\section{Physical examination}

Physical examination of patients who experience $\mathrm{PE}$ is often unremarkable. Considering that PE may co-exist with other sexual dysfunctions, especially erectile dysfunction (18), the International Society for Sexual Medicine (ISSM) strongly recommends performing a physical examination in these cases (7). For lifelong PE, some patients found it reassuring for the physician to examine them. Regarding acquired $\mathrm{PE}$, a targeted physical examination helps to rule out any acute or chronic illness or injury that may contribute to ejaculation dysfunction. These illnesses include urinary and reproductive tract infections, Peyronie's disease, neurological impairment, and endocrine dysfunction (19). However, there are no specific criteria to confirm or exclude PE. The main purpose of the physical examination is to distinguish PE from ED or to rule out any underlying diseases or conditions that may cause PE.

\section{Patient-reported outcome (PRO) questionnaires}

Since it is difficult to determine whether a man has PE based on the patient history and physical examination, there is an apparent need for a valid and reliable screening instrument for PE. Rowland et al. (20) first utilized 2-question diagnostic utility based on the Diagnostic and Statistical Manual of Mental Disorders, Fourth Edition, Text Revision (DSM-IV-TR) definition of PE for diagnosis in 2004. However, the decision of whether a particular question should be asked in each clinical session relies mainly on the physician. This situation may lead to varied sets of questions applied to the same patient and, consequently, result in inconsistent diagnoses by different physicians. Therefore, standardized questionnaires and surveys are proposed with the aim to evaluate subjective symptoms and perform comprehensive data collection. Currently, the index of premature ejaculation (IPE), the premature ejaculation diagnostic tool (PEDT), and the premature ejaculation profile (PEP) are the questionnaires that are applied most frequently in a clinical setting and that meet most of the criteria for test development and validation (21). They provide reliable, interpretable, and standardized assessment measures for the diagnosis and differentiation diagnosis of PE. Other self-reported questionnaires, including the Chinese Index of Premature Ejaculation (CIPE) (22) and the Arabic Index of Premature Ejaculation (AIPE) (23), are not recommended for clinical use due to the lack of adequate clinical data or not meeting the DSM-IV-TR criteria $(9,21)$. The features, advantages, and limitations of these questionnaires are shown in Table 2.

\section{IPE}

The IPE questionnaire was developed and validated by Althof et al. in 2006 (8). It consists of 10 items in 3 domains for the assessment of PE-related issues, including control over ejaculation, satisfaction with sex life, and level of distress in men with this condition. The IPE questionnaire has been shown to correlate well with the IELT and have good known-groups' validity and testretest reliability. Compared to other valid questionnaires, the most apparent feature of the IPE is that it covers detailed core features of the overall experience of PE, thus providing a more comprehensive assessment. It is also relatively brief and easy to administer. Based on these advantages, IPE has been used to assess the outcome of PE treatment in clinical trials (24). However, lacking grading norms and diagnostic cut-off values can make the reassessment results used to evaluate improvement versus deterioration of the PE condition rather ambiguous, hence more difficult to determine treatment. Additionally, as DSM-5 has updated the definition of PE and limited the IELT to approximately $1 \mathrm{~min}$, the DSM-IV-TR based IPE may need further modification and validation to adapt to the new definition.

\section{PEDT}

In determining the presence or absence of PE, Symonds et al. developed the PEDT questionnaire in 2007 and made this task valid and straightforward (9). This questionnaire consists of 5 items that meet the DSM-IV-TR criteria and assesses ejaculatory problems, such as aspects of ejaculatory control, frequency of sexual intercourse, minimal sexual stimulation, distress, and interpersonal difficulty. Each question is rated on a scale ranging from 0 to 4 , and an index score is derived from the sum of the responses to 
Table 2 Features of clinic diagnose tool for premature ejaculation

\begin{tabular}{|c|c|c|c|c|c|c|}
\hline Method & $\begin{array}{l}\text { Number of } \\
\text { questions }\end{array}$ & Domain names & Advantages & Defects & $\begin{array}{l}\text { Evaluation } \\
\text { period }\end{array}$ & $\begin{array}{c}\text { Clinical } \\
\text { applications }\end{array}$ \\
\hline $\begin{array}{l}\text { ISSM } \\
\text { Recommended } \\
\text { Enquiry }\end{array}$ & Multiple & $\begin{array}{l}\text { PE diagnose; PE phenotype; } \\
\text { Erectile function; } \\
\text { interpersonal relationship } \\
\text { assess; previous treatment; } \\
\text { impacts on life }\end{array}$ & $\begin{array}{l}\text { Causal in chat; } \\
\text { comprehensive } \\
\text { assessment; easy to use; } \\
\text { oral presentation only }\end{array}$ & $\begin{array}{l}\text { Lack of scoring } \\
\text { system; optional } \\
\text { questions; easy to } \\
\text { omit items; variable } \\
\text { to administer }\end{array}$ & Non-defined & Yes \\
\hline $\begin{array}{l}\text { Index of } \\
\text { Premature } \\
\text { Ejaculation }\end{array}$ & 10 & $\begin{array}{l}\text { Control over ejaculation; } \\
\text { sexual satisfaction; PE } \\
\text { personal impact; PE } \\
\text { interpersonal impact }\end{array}$ & $\begin{array}{l}\text { Easy to use; standardized } \\
\text { options; defined grading } \\
\text { for subjective item; quantile } \\
\text { grading system; exit strategy; } \\
\text { multiple questions for each } \\
\text { domain }\end{array}$ & No Scoring system & 4 weeks & Yes \\
\hline $\begin{array}{l}\text { PE Diagnostic } \\
\text { tool }\end{array}$ & 5 & $\begin{array}{l}\text { Control over ejaculation; } \\
\text { self-perception evaluate; } \\
\text { PE personal impact; PE } \\
\text { interpersonal impact }\end{array}$ & $\begin{array}{l}\text { Scoring system; quartile } \\
\text { grading system; } \\
\text { quick assess; } \\
\text { defined grading }\end{array}$ & $\begin{array}{l}\text { No exit strategy } \\
\text { mostly subjective } \\
\text { description; single } \\
\text { question for domains }\end{array}$ & Non-defined & Yes \\
\hline $\begin{array}{l}\text { Chinese Index } \\
\text { of Premature } \\
\text { Ejaculation }\end{array}$ & 10 & $\begin{array}{l}\text { Sexual libido; erection } \\
\text { respond; estimated IELT; } \\
\text { control over ejaculation; } \\
\text { sexual satisfaction; PE } \\
\text { interpersonal impact; PE } \\
\text { personal impact }\end{array}$ & $\begin{array}{l}\text { Easy to use; standardized } \\
\text { options; penile erectile } \\
\text { evaluation; IELT access; } \\
\text { Quinte grading system (ten } \\
\text { for IELT assess); scoring } \\
\text { system }\end{array}$ & $\begin{array}{l}\text { Undefined scoring } \\
\text { strategy; undefined } \\
\text { exit strategy; single } \\
\text { question for domains }\end{array}$ & Non-defined & Not given \\
\hline
\end{tabular}

the 5 questions. Since there are only a few questions that patients need to answer, clinicians can quickly identify whether a "probable PE" exists in a patient. By employing a 3 -tiered cut-off score, the PEDT classifies cases as PE $(\leq 8)$, possible $\mathrm{PE}$ (9 or 10$)$, and absence of $\mathrm{PE}(\geq 11)$.

Moreover, the score is indicative of the need for further treatment in each case. For possible PE, clinicians need further investigation to determine the existence of dysfunction. However, it should be noted that PEDT is not suitable for evaluating the PE treatment efficacy or indicating a designed intervention, because this scoring system is not purposely designed with this objective. Meanwhile, similar to the IPE, the PEDT may also require a re-validation to adapt to the DSM-5 criteria.

\section{PEP}

The PEP is a 4-item PE assessment tool, developed and validated by Patrick et al. in 2009, that measures all domains of ejaculatory function as defined by the DSM-IV-TR: control over ejaculation, satisfaction with sexual intercourse, interpersonal difficulties, and distress related to ejaculation (10). Each question is rated with a scale ranging from 0 to 4 , and higher scores indicate better function. An index score is calculated by averaging the sum score of the four specific PEP questions. This questionnaire is concise, easy to administer, and has good test-retest reliability and knowngroups' validity. The PEP has been used to evaluate the efficacy of Dapoxetine (25) and PSD-502 (24) in treating 
men with $\mathrm{PE}$ in clinical trials, as well as to characterize $\mathrm{PE}$ in observational studies (26). However, its simplicity is also its weakness, and its reliability is a concern. For example, the result of 1 domain of PE is determined solely with a single question (27). Theoretically, it is difficult to cover all men's issues concerning PE with four questions, regardless of how well the four questions were designed. Another concern regarding the PEP is that there are no studies to further verify its validity and reliability based on the new DSM-5 diagnostic criteria for PE proposed by the ISSM, which resembles the IPE and the PEDT. Additionally, the lack of validated cut-off scores makes the PEP more suitable for clinical research rather than clinical practice.

Taken together, these validated PRO questionnaires serve as useful tools to assess PE for family practice physicians and sexual medicine experts. However, the discontinuity among these tools may restrict their applicability in clinical practice, since the data collected by each tool is non-unified and unconvertible. Further, the lack of cut-off scores on some questionnaires (IPE and PEP) makes it difficult for physicians to judge whether a patient has PE when solely relying on these measures. In a study conducted in Korea, the researchers found that the rate of application of these diagnostic tools is merely $42 \%$ in clinical practice, and clinicians are more inclined to make the PE diagnosis based on their patients' subjective descriptions (28). In this regard, the combination of 2 to 3 validated questionnaires, such as the PEDT combined with the IPE or PEP, may be a feasible solution to improve $\mathrm{PE}$ assessing accuracy.

Moreover, it is important to realize that these measures cannot replace a detailed sexual history obtained by a professional clinician and can only serve as useful adjuncts (7). Recently, a new unpublished patient-reported outcome tool, the Patient Outcome for Premature Ejaculation (POPE), has been completed by the Ampio Pharmaceuticals and accepted by the FDA for the evaluation of PE therapy in the United States [Available at: https:// adisinsight.springer.com/trials/700219252 (Date of access 7/10/2018) (2013)]. It is a revision of the PEP, in which the wording of the distress question is modified, and the three remaining questions are unaltered (27).

\section{Ejaculation latency time}

\section{Establishment of the IELT}

Despite assessment of the domains of control over ejaculation, sex satisfaction, and negative personal impact are recommended in the diagnosis of $\mathrm{PE}$, currently $\mathrm{PE}$ diagnosis is highly relying on the IELT calculation. IELT is the time latency for ejaculation to occur, starting from the first penile-vaginal penetration (11). The first introduction of IELT was conducted by Waldinger et al. while studying paroxetine's effects on PE treatment (11).

In the previous DSM criteria, no cut-off point for the ejaculatory latency was defined (29). Waldinger et al. assessed the ejaculatory latency in men with lifelong PE using a stopwatch and identified that the latency within 1 minute is manifested in $85 \%$ patients (30). Using the same methods, subsequent studies confirmed that 3 minutes is an optimal cut-off value for the diagnosis of acquired PE $(31,32)$. These works clarified previous ambiguity in $\mathrm{PE}$ definition and revolutionized it by redefining the $\mathrm{PE}$ classification (33). Meanwhile, Waldinger and Serefoglu respectively identified the "variable" and "subjective" as the new PE categories to classify patients whose IELT is either undefinable or longer than the other two types of PE (34). Later, in the DSM-5, ISSM further updated the definition of the ejaculatory latency value as "approximately 1 minute following vaginal penetration" by adding "before the person wishes" (4). Definitive IELT values optimized the uniformity in clinical application and treatment research (35). Identifying the PE category was facilitated by the IELT, which is assessed by a stopwatch or is based on the patient's estimation, since it provides a more plausible and definitive index to fulfill the preliminary screening (36). It has become a more prevalent index in PE research.

\section{Limitations in intravaginal ejaculation latency time}

Patrick et al. analyzed $408 \mathrm{PE}$ versus 2,294 non-PE individuals by pooling them into tertile subgroups according to their IELT. The result revealed that the IELT tertile subgroups ideally manifested patients' reported global impression and change in their ejaculatory problem. However, in their survey, the 1 min IELT criterion limited the inclusion of PE subjects (10). Also, data from other investigations revealed a vast overlap in IELT distribution between PE and non-PE subjects. This overlap is non-neglectable, although IELT was significantly shorter in PE (37).

Besides, the reliability of reported IELT was questioned. In Pryor's research, the estimated and calculated IELT in males with or without PE was compared, and they concluded that while IELT was objectively correlated with PE severity, these patients were more inclined to overestimate their IELT to a greater extent (38). Their research revealed the tendency of overestimating IELT. Besides, the perceptive disparity between patients and their partners also should be noted (39). Patrick et al. pointed out that due to 
personal distress and psychological burdens, PE patients are more likely to resolve their problem by reporting an "estimated normal IELT" instead of seeking medical help. The estimated normal IELTs were 7 to 13 minutes, which is significantly longer than their partners (39). Their result was contradicted with Cal's research; however, it is confirmed by the research performed by Rosen et al. $(40,41)$, which suggests that combining patients reported outcome measurement might improve IELT validity in PE assessment and outcome (40).

Furthermore, IELT defines the penile intromission solely as intravaginal. It is non-neglectable that disparities in the penile thrust tracks and vaginal stress tone influenced on ejaculations (42). In Bhat's investigation, the ejaculatory latency time was $240 \pm 38.6$ seconds to $700 \pm 79.1$ seconds, depending on different sexual stimulation (43). This result suggests that intensity and total amount of penile stimulation before ejaculation is variable. Nonetheless, IELT is inadequate in evaluating ejaculatory latency other than penovaginal contact. In noticing this limitation, Waldinger proposed the MELT, OELT, and AELT to assess ejaculatory latency regarding the aspects of masturbation, oral sex, and anal sex (44).

Additionally, IELT should be based on a calculation that starts at the first penovaginal intromission, whereas intercourse includes activities such as foreplay and change of coitus position. The demand for the first intromission is rather challenging to fulfill, not to mention that the use of a stopwatch is intrusive. Indeed, it may aggravate the PE problem due to a negative personal impact by disrupting sexual spontaneity and intercourse fluency (45).

These results suggest that by basing PE assessment on ejaculation latency, males were deemed as the sole culprit. However, it is advisable to consider and appraise the influence from both sexual partners, as well as consider the need for additional measures to characterize PE (39).

\section{Penile sensory evaluation}

Penile hypersensitivity was believed to be a significant factor in PE (46). Biothesiometry, or vibrometers, are devices that are capable of producing vibrations with different amplitudes. It was initially designed to assess abnormal afferent somatic pathways and peripheral neuropathies and subsequently was introduced in penile sensitivity evaluation (46). Technically, to assess penile sensitivity, a vibratory stimulator is required to sweep over the penis, including at the sites of the glans penis and the penile shaft. Also, this sensory threshold should be rectified with a synchronized hand vibrator on a peripheral site, mostly at the index finger (47). Thus, it is believed that the vibrometers would be able to provide objective penile sensory information, thereby facilitating $\mathrm{PE}$ diagnosis.

\section{Controversies in penile biothesiometry research}

Initial investigation on penile sensitivity with vibrating devices dates back to the 1970s (12). However, to date, these findings add more confusion to the interpretation of PE etiology. In revealing PE etiology, early research has both validated and disproved the existence of penile hypersensitivity $(48,49)$. Furthermore, the correlation between IELT and penile sensory threshold was investigated by several studies with contradictory results $(50,51)$. Interestingly, some research further revealed that the penile sensory thresholds were even higher in patients with PE, which contradicts the notion that penile hypersensitivity induces PE $(51,52)$. These facts revealed a significant disparity in the field of penile biothesiometry research.

In the research studies conducted by Xin and Paick, the penile sensory threshold was compared between $\mathrm{PE}$ patients and potential PE patients $(48,53)$, whereas in Guo and Vanden Broucke's research, the selected subjects were labeled as either non-PE or PE for each assessment (50,54). In the latter research, the results only elucidate the features of penile sensitivity in "normal" and "abnormal" subjects. Although these authors attempted to decipher the penile sensory threshold in individuals with different ejaculatory thresholds, it is unclear whether these results are applicable in interpreting PE etiology.

The concomitance of ED and PE is prevalent. In some research, the potential effect of ED and neuropathology was excluded, and the PE criteria were defined as ejaculation latency less than $1 \mathrm{~min}$. Nevertheless, Rowland included subjects with primary PE, secondary PE, ED concomitant, ED patients, and patients without impotence in their investigation (51). It is clear that these researchers attempted to elucidate sexual dysfunction but, given that the etiology of both dysfunctions remains unclear, involving ED increases the complexity in comprehending the results of the study. Furthermore, it is noteworthy that the time when this research was conducted predates the introduction of the latest PE classification criteria. Thus, the yet-to-be optimized selection may potentially compromise the results.

Additionally, while both research studies performed by Xin and Paick investigated penile sensitivity in PE subjects, Xin enrolled 120 subjects, whereas Paick enrolled 33 subjects. The mean age gaps were inconsistent between 
the groups in their investigations $(48,53)$. This indicated that inconsistent subjects' selection criteria and limited sample size cannot rule out the potential selection bias and may undermine research validity. Also, Paick mentioned performing an intra-cavernous injection (53) in their research, while such operation was not mentioned in other research. This revealed an absence of standard operating procedure in guiding biothesiometry research. These facts may partially explain the disparities observed between these 2 studies.

\section{Disparity in the investigation protocol}

In the researches mentioned above, the settings of each vibrometer ensured that control of vibration intensity is subjective and facilitated. In these studies, even though all tests were performed by the same technician, the operational consistency in each test is subject to manual operations. Although the vibrating scales are within the range of micrometers in equipment, a manual operation seems difficult to perform in each test (52). This fact reveals a vague correlation between "vibration intensity" and penile sensitivity, and that the technique in studying penile sensitivity is probably inadequate.

First, future research should focus on the penile erectile property. In research studies performed by Xin and Rowland, penile sensory thresholds were evaluated with the flaccid penis $(48,51,55)$. Meanwhile, Vanden Broucke et al. tested vibrating stimulations on the stretched and flaccid penis (50), whereas, Paick et al. evaluated penile sensitivity on the flaccid and erected penis (53). Though the difference was insignificant, the penile sensitivity tends to be higher when the penis was erected, especially in premature subjects. This result was further confirmed by Hill et al., who specifically investigated the correlation between penile erectile property and its sensitivity (56). Additionally, the maneuvers were either "performed by the same operator" or "attached the penis with the equipment on its own weight". These states suggest that inconsistent requirements of erectile properties, combined with operation differences potentially underlies the heterogeneity in studies.

Still, the practical constraints of applied biothesiometers, especially the probe shape, were different between studies. Despite the range of vibration scales and the fact that the intensity produced in each device is similar and comparable, the disparity of penis-probe contacts in each investigation resulting from equipment design is non-neglectable $(52,56)$, which has been described as "contacting with probe" or "resting of the device on its own weight" in each study.
Notably, in the research performed by Vardi, the device was designed and referred from an investigation on a study evaluating the sensitivity of female genitalia (57), making probe-penis contact impossible. To solve this problem, they had to "attach an additional probe" to isolate the vibration away from the weight of the integrated equipment. Consequently, these facts imply that although the investigator can at least ensure operational consistency in their own study, the parameter and the result may not be translatable in different studies.

A similar operational difference is also manifested in the protocols in each type of research. In aiming to decipher the correlation between vibration intensity and penile hypersensitivity, Guo et al. applied a fixed frequency with various vibrating amplitudes in their protocol. Though a similar technique was applied in the research of Chen and Vanden Broucke, the vibrating frequencies of their stimulation were different, ranging from 80 to $120 \mathrm{~Hz}$ $(49,50)$. This fact makes the vibrating frequency a new variant. Again, in Salonia's investigation, they included the thermal stimulus in their investigation (52). While their findings revealed that the penile sensitivity in PE patients was variable in detecting "cold" stimulations, their results only highlight that it is not a "de-sensitivity" but "hypersensitivity" status in PE subjects (52).

In addition, as an examiner of peripheral sensory neuropathy, the stimulus of biothesiometry should be rectified with reference peripheral portion, typically at the index finger or finger pulp (46). Other referential sites, such as wrist, ankle, or right-hand palm, were applied in other investigations as well $(49,51)$. It is suggested that the vibration perception threshold (VPT) provides important and meaningful information about nerve fiber dysfunction, and the proposed testing site is at the index finger or the first hallux (46). Thus, there is no evidence that testing other peripheral parts are equally valid as a reference point for the index finger.

\section{Limitations in the penile biothesiometry test}

There are limitations to stimulation innervated by vibrometers. First, it focuses solely on specific surface areas on the penis, while the coital stimulating pattern on the penis is yet to be determined. It is reasonable to believe that coital penile stimulation is more complicated and comprehensive than the single or multiple probes that require a piece of equipment. Meanwhile, Paick et al. argued that there was evidence that a programed vibrating pattern is adequate to induce ejaculation (53). Nevertheless, 
there are specific limitations in simulating coital stimulation with a vibrometer on the penis. Further efforts are needed to assess penile somatosensory innervation and to determine penile sensitivity by current vibrating innervation. Also, it was reported that anxiety affects patient's ejaculation, as well as their perception sensitivity $(58,59)$. It is reasonable to assume that patients' sensory threshold is possibly affected by their consciousness and attentiveness during the sensory test.

In all, it has been decades since biothesiometry was first introduced to test penile sensation and diagnose PE. Currently, available results may seem controversial, but it is still one of the few approaches that provide subjective information about penile peripheral neurotransmission features. Meanwhile, penile hypersensitivity is still believed to be the main cause of some types of PE (60). Biothesiometry is still a promising method since it identifies the correlation between penile sensitivity and PE; however, certain amendments in operational technique and testing protocol should be performed to guarantee a plausible and interpretable result. The examination protocols, characteristics, and results of the aforementioned penile biothesiometry studies are shown in Table 3 .

\section{Penile electrophysiological test}

Based on the notion that penile hypersensitivity is one of the leading causes of $\mathrm{PE}$, the penile electrophysiological test was introduced in order to decipher the link between the neurological factor in penile hyperexcitation and primary PE (13).

The main electrophysiological tests introduced in PE diagnosis were the somatosensory evoked potentials (SEPs) and the bulbocavernosus reflex potentials (BCPs). To measure a penile somatosensory-related SEP, a stimulus electrode was placed on the penile shaft and glans penis and a recording electrode placed on the midline scalp and the electroencephalographic recording sites $(60,61)$. The objective of this method is to give a cortical representation of the sensory stimuli arising from the genital area to the primary or secondary somatosensory cortex (S1 and S2) in the cerebral cortex (61).

\section{Mechanism of SEP and BCP}

Numerous types of SEP tests were used since their initial application. Currently, the main types of SEPs include somatosensory evoked potential of pudendal nerve (PNSEP), somatosensory evoked potential of dorsal nerve (DNSEP), and somatosensory evoked potential of the glans penis (GPSEP) (62). PNSEP is designed to evaluate the complete peripheral and central pudendal nerve afferent pathway of the perineal region, whereas the DNSEP and GPSEP were the optimized methods aimed at specifically assessing the neurophysiological properties of penis innervation (61). Although it is recommended that the stimuli should be applied at the penile shaft and glans penis when measuring these SEPs (63), Xin et al. pointed out that the accurate SEPs could not be recorded by stimulating the glans penis (64). Hence, he suggests that a proper modification should be made during the experiment to ensure a plausible result. Accordingly, lacking the standard of criteria and operational procedure is potentially introducing variability in SPE measurement in different research studies.

The bulbocavernosus reflex (BCR) and bulbocavernosus motor evoked potentials (BC-MEP) are designed to evaluate the afferent and efferent conduct through the pudendal nerve at the S2-S4 spinal segment (65). During the ejaculation process, an involuntary contraction over the genital-anal region is indicating the commencement of seminal expulsion. This response is easily recorded by BCR monitoring; hence, it is suggested that the BCR potential provides innate information on the primary penile conducting properties and its response to the innervating stimulations (66). To measure the BCR potential, two electrodes were placed on the distal shaft of the penis and the bulbocavernosus muscles (64).

\section{Penile electrophysiological findings in PE}

SPEs mainly provide a record of cortical and local response to sensory stimulations. In investigating PE patients, Fanciullacci et al. discovered that their SEP amplitude was statistically higher than the control group (67). This result revealed that penile hypersensitivity is a precursor to $\mathrm{PE}$ (67). Other research investigating PE patients further confirmed this conclusion with similar results $(64,68)$. Moreover, experiments on both humans and animals revealed that local penile anesthesia reduces SEP amplitude stimulation at the glans penis and prolongs its latency $(69,70)$. However, these results were contradicted with the results obtained by Perretti et al. (71). Similarly, in the investigation of SEPs in patients characterized with delayed ejaculation, a situation considered opposite to PE, Xia et al. discovered that these patients were characterized by penile hyperexcitability (72). These findings make the penile SEP test more perplexing.

Regarding the BCP, Zhou and Jiang discovered that the BCR threshold was significantly lower in PE patients 
Table 3 Results of penile biothesiometry finding

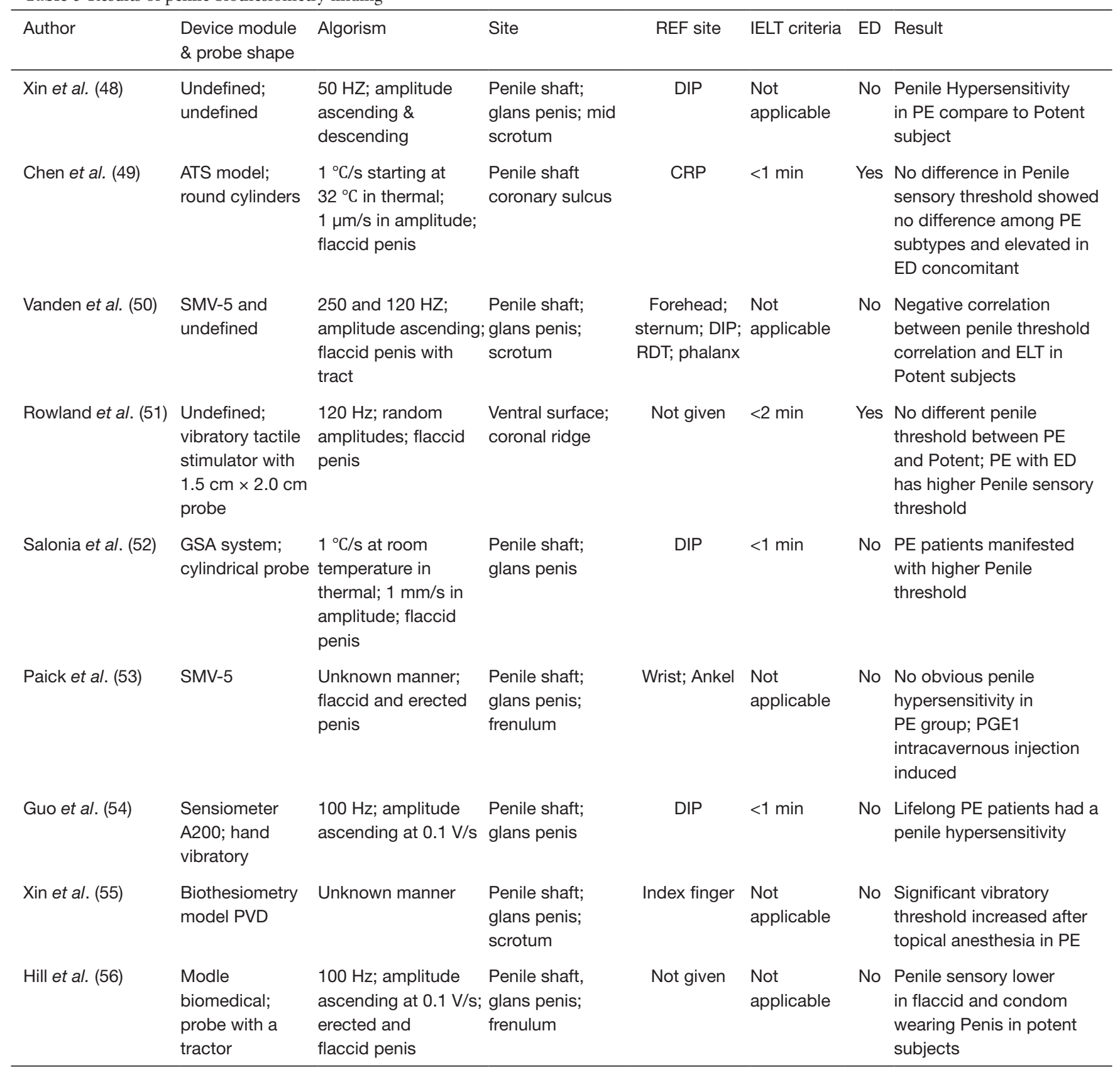

$\mathrm{PE}$, premature ejaculation; ED, erection dysfunction; DIP, dominant index finger pulp; RDT, right distal toe; CRP, center of right palm. 
compared with the control group $(66,73)$. However, their result was contradicted with Perretti's research (71). Hence, it is evident that disparities still exist in penile neurophysiology examination due to certain limitations.

\section{Limitations in penile electrophysiological test}

Recently, Gao et al. discovered positive structural abnormalities in the neural conduction pathways in PE patients (74). This finding confirmed the altered conductivity in the electrophysiological exams. However, the correlation between penile hypersensitivity and $\mathrm{PE}$ due to the hyperexcitable penile glans is merely partially confirmed, and accelerated peripheral conduction has only been proven by some research studies (60). It is presumed that several factors, such as age, height, gender, body temperature, site of stimulation, type of nerve fiber, degree of myelinization, and nerve pathway, may predispose to SPEs (64). Therefore, a more effective screening mechanism of the subjective and standardized operating process with a unified index is required in performing electrophysiological tests. The examining protocols, characteristics, and results of aforementioned penile electrophysiological investigations are shown in Table 4.

Currently, available $\mathrm{PE}$ research using penile electrophysiological is less frequent, and the studies investigating $\mathrm{PE}$ using this technique focus mainly on patients with primary ejaculatory dysfunctions. This may be because electrophysiological techniques are mainly suitable for monitoring the primary cortex and peripheral neural pathways at lower levels, while it is easier to rule out correlated and unidentified etiological conditions in patients with primary ejaculation dysfunctions (64). In the investigation carried out by Yilmaz, it was revealed that patients' penile sensory threshold and SEPs remained unchanged, although fluoxetine alleviates the patients' premature disorder by prolonging their IELT (65). In this case, it is suggested that the electrophysiological test only partially illustrates the penile sensory properties and is influenced by a variety of factors. Due to these limitations and controversies, the SPE and BCP are both applicable in providing neurophysiological data of subjects; however, their validity in determining penile hypersensitivity and in clarifying PE etiology is yet to be verified.

\section{Discussion}

Accurate PE evaluations have relied on previous ambiguous definitions and unvalidated examination techniques. Clinical diagnosis and research are mainly based on patient history, patient-reported outcome scores, and diagnosis tools. Undoubtedly, these diagnostic methods provided valuable information regarding the PE condition and the related negative personal consequences; however, there is a lack of feasible and plausible means of objective measurement to evaluate the ejaculatory latency and control over ejaculation.

In the meantime, calculating ejaculatory latency time, especially IELT, is becoming increasingly critical in evaluating and describing ejaculatory disorders. This index emphasizes calculation of the temporal dimension during penile thrusting before ejaculation, but it is noteworthy that this indicator tends to interpret and measure $\mathrm{PE}$ as an absolute objective and quantifiable behavior, which it is not. Namely, "intravaginal" thrust is one of the main patterns of penile stimulation to induce ejaculation. It is possibly not the only means that is involved in the actual coital process (75), even though the duration calculation is potentially biased due to compromised standard operating procedures. Hence, ejaculatory latent time provides a sophisticated but comprehensive indication for interpretation of the ejaculatory condition, not just as an outcome.

In simulating penile stimulation, the penile biothesiometry and electric pulses seem plausible. However, the current stimulating pattern was restricted in location and vibration or electrical pattern. This is because this technique continues to focus on measuring penile sensory level and deciphering penile hypersensitivity. It is unknown whether this notion is indicative of PE etiology, but vibrating stimulation does not simulate or resemble actual coitus sensation. In other words, the "vibration" does not resemble "thrusting." Even though the vibrating strengths and frequency are readily presented and adjustable in each test, the "sensory threshold" is ideally presented as a vibrating or electrical stimulation value.

Penile stimulation substantially underlies the ejaculatory process. Multiple levels of motor-sensory reflexes are involved in the ejaculatory process, including penile erection, emission, chamber pressure formation, and sperm expulsion (76,77). As been described by Herbert, the maintenance of erectile status and ejaculation latency is dominantly innervated and modulated by stimulating the penis through thrusts (78). Also, he further demonstrated that in the pudendal nerve tampered rhesus monkeys, more intensive and prolonged penile thrusting is required to achieve ejaculation (78). He believed that because the neural pathways had been partially intersected, more penile stimulation was required to achieve a "sufficient" signal to trigger the expulsion phase of ejaculation. So, the increased 
Table 4 Results of penile electrophysiological finding

\begin{tabular}{|c|c|c|c|c|c|}
\hline Author & Potential type & Protocol & Stimulation location & IELT criteria & Result \\
\hline Xia et al. (61) & $\begin{array}{l}\text { GPSEP; } \\
\text { DNSEP }\end{array}$ & $\begin{array}{l}1.0 \mathrm{~ms} \text { at } 3 \mathrm{~Hz} ; 10.0 \mathrm{~mA} \\
\text { for stimulation; ascending } \\
\text { descending to minimal } \\
\text { perception for threshold }\end{array}$ & $\begin{array}{l}\text { Subcoronal-DN; } \\
\text { glans penis-GP }\end{array}$ & $<3 \mathrm{~m}$ in $\mathrm{PE}$ & $\begin{array}{l}\text { GNSPE latency prolonged } \\
\text { with IELT prolongation after } \\
\text { circumference }\end{array}$ \\
\hline Xin et al. (64) & $\begin{array}{l}\text { DNSEP; } \\
\text { GPSEP }\end{array}$ & $\begin{array}{l}0.05 \mathrm{~ms} \text { at } 3 \mathrm{~Hz} \text {; from } 0 \text { to } \\
80 \text { to } 100 \mathrm{~V}\end{array}$ & $\begin{array}{l}\text { DN-penile shaft; } \\
\text { GP-glans penis }\end{array}$ & Not given & $\begin{array}{l}\text { PE patients showed higher } \\
\text { DN-GN-SEP amplitude }\end{array}$ \\
\hline Yilmaz et al. (65) & $\begin{array}{l}\text { Cortical SEP; } \\
\text { sacral ER }\end{array}$ & $\begin{array}{l}0.2 \mathrm{~ms} \text { at } 2 \mathrm{~Hz} \text {; ascending } \\
\text { descending to minimal } \\
\text { perception }\end{array}$ & $\begin{array}{l}\text { DN-GP_subcoronal } \\
\text { region and shaft }\end{array}$ & Not given & $\begin{array}{l}\text { No different SEP and latency } \\
\text { change while IELT and penile } \\
\text { sensory threshold elevated }\end{array}$ \\
\hline $\begin{array}{l}\text { Fanciullacc et al. } \\
(67)\end{array}$ & Cortical SEP & $0.2 \mathrm{~ms}$ at $3 \mathrm{~Hz}$ & Genital area & Not given & $\begin{array}{l}\text { True PE manifested higher } \\
\text { SEP representative from } \\
\text { genital area }\end{array}$ \\
\hline Yang et al. (68) & $\begin{array}{l}\text { DNSEP; } \\
\text { GPSEP; } \\
\text { PSSR }\end{array}$ & $\begin{array}{l}1.0 \mathrm{~ms} \text { at } 3 \mathrm{~Hz} \text {; ascending } \\
\text { descending to minimal } \\
\text { perception for threshold }\end{array}$ & $\begin{array}{l}\text { Subcoronal-DN; } \\
\text { glans penis-GP }\end{array}$ & $<2 \mathrm{~m}$ in $\mathrm{PE}$ & $\begin{array}{l}\text { PE patient with shorter } \\
\text { latency than potent subjects }\end{array}$ \\
\hline
\end{tabular}

PE, premature ejaculation; DE, delayed ejaculation; MEP, motor evoked potential; Sacral ER, sacral evoked response; PSSR, penile sympathetic skin response; DN, dorsal nerve; GP, glans penis.

amount and intensity of penile thrusts are the requirement for "sufficient stimulation" to induce ejaculation.

Although attempts at calculating penis thrust before ejaculation dates back to the 1970s (79), this criterion seemed to never enter into the mainstream since its reliability and accessibility were questioned, and it was subsequently substituted by the introduction of IELT (80). A conspicuous defect was that the focus was solely on the thrusting numbers, but omitted the parameters of thrust frequency, depth, and pausing intermission during thrusts-the typical scenes in real coitus. These cofactors are also significantly contributing to the penile stimulations. Other factors are affecting the ejaculation process. For example, $1 \mathrm{PE}$ patient can withhold a single thrust during intravaginal intercourse and can wait for the second thrust after the ejaculation impulse has ceased in order to increase his pre-ejaculation thrust numbers and, consequently, the latency time. 
Diagnosing $\mathrm{PE}$ is as complicated a task as finding an accurate definition for this condition. Considerable progress has been achieved in amending PE diagnostic guidelines and improving the PRO questionnaire. IELT provides a subjective index in PE description, but its accuracy and definiteness are yet to be validated. Based on the penile biothesiometry, PE assessments and parameters should be designed by referring to the natural course of penile thrust to justify ejaculator disorders. However, instead of calculating latency time or thrust number, the ejaculatory threshold should be appraised. In this case, determining the threshold requires calculating the stimulatory duration and scaling its magnitude along with its changing pattern. These data could be combined with data on the stimulatory intensity and frequency that the penis has endured before ejaculation, thus allowing for the evaluation and quantification of the total stimulus required to accomplish ejaculation.

\section{Conclusions}

Currently, available data on psychogenic or neurogenic etiology of $\mathrm{PE}$ are controversial and inconclusive. Present methods in PE assessment revolutionized PE research and allowed for a greater understanding of this condition. However, a single parameter is not enough to describe and quantify PE. Comprehensive penile stimulation simulates real sexual intercourse, and the intensity and duration parameters recorded before the ejaculatory threshold are more objective relative to a single chronological observation when describing and diagnosing PE.

\section{Acknowledgments}

Funding: This paper was supported by the Natural Science Foundation of China (No. 81671453, 81270691, and 81170565). Sichuan Science and Technology Program No. 19RKX0475 and Thousand Talent Plan of Sichuan Province.

\section{Footnote}

Conflicts of Interest: All authors have completed the ICMJE uniform disclosure form (available at http:// dx.doi.org/10.21037/tau.2019.12.08). FQ and JY have a patent INTELLIGENT MONITOR OF ERECTILE FUNCTION issued. The other authors have no conflicts of interest to declare.
Ethical Statement: The authors are accountable for all aspects of the work in ensuring that questions related to the accuracy or integrity of any part of the work are appropriately investigated and resolved.

Open Access Statement: This is an Open Access article distributed in accordance with the Creative Commons Attribution-NonCommercial-NoDerivs 4.0 International License (CC BY-NC-ND 4.0), which permits the noncommercial replication and distribution of the article with the strict proviso that no changes or edits are made and the original work is properly cited (including links to both the formal publication through the relevant DOI and the license). See: https://creativecommons.org/licenses/by-nc$\mathrm{nd} / 4.0 /$.

\section{References}

1. Saitz TR, Serefoglu EC. The epidemiology of premature ejaculation. Transl Androl Urol 2016;5:409-15.

2. McMahon CG, Lee G, Park JK, et al. Premature ejaculation and erectile dysfunction prevalence and attitudes in the Asia-Pacific region. J Sex Med 2012;9:454-65.

3. Gao J, Peng D, Zhang X, et al. Prevalence and Associated Factors of Premature Ejaculation in the Anhui Male Population in China: Evidence-Based Unified Definition of Lifelong and Acquired Premature Ejaculation. Sex Med 2017;5:e37-e43.

4. McMahon CG, Althof S, Waldinger MD, et al. An evidence-based definition of lifelong premature ejaculation: report of the International Society for Sexual Medicine Ad Hoc Committee for the Definition of Premature Ejaculation. BJU Int 2008;102:338-50.

5. Kalejaiye O, Almekaty K, Blecher G, et al. Premature ejaculation: challenging new and the old concepts. F1000Res 2017;6:2084.

6. Serefoglu EC, Saitz TR. New insights on premature ejaculation: a review of definition, classification, prevalence and treatment. Asian J Androl 2012;14:822-9.

7. Althof SE, McMahon CG, Waldinger MD, et al. An update of the International Society of Sexual Medicine's guidelines for the diagnosis and treatment of premature ejaculation (PE). J Sex Med 2014;11:1392-422.

8. Althof S, Rosen R, Symonds T, et al. Development and validation of a new questionnaire to assess sexual satisfaction, control, and distress associated with premature 
ejaculation. J Sex Med 2006;3:465-75.

9. Symonds T, Perelman MA, Althof S, et al. Development and validation of a premature ejaculation diagnostic tool. Eur Urol 2007;52:565-73.

10. Patrick DL, Giuliano F, Ho KF, et al. The Premature Ejaculation Profile: validation of self-reported outcome measures for research and practice. BJU Int 2009;103:358-64.

11. Waldinger MD, Hengeveld MW, Zwinderman AH. Paroxetine treatment of premature ejaculation: a doubleblind, randomized, placebo-controlled study. Am J Psychiatry 1994;151:1377-9.

12. Newman HF. Vibratory sensitivity of the penis. Fertil Steril 1970;21:791-3.

13. Vignoli GC. Premature ejaculation: new electrophysiologic approach. Urology 1978;11:81-2.

14. Porst H, Montorsi F, Rosen RC, et al. The Premature Ejaculation Prevalence and Attitudes (PEPA) survey: prevalence, comorbidities, and professional help-seeking. Eur Urol 2007;51:816-23; discussion 824.

15. Lee G, McMahon CG, McCabe M, et al. Initiators and Barriers to Discussion and Treatment of Premature Ejaculation Among Men and Their Partners in Asia Pacific - Results From a Web-based Survey. Sex Med 2016;4:e233-e41.

16. Moreira ED Jr, Kim SC, Glasser D, et al. Sexual activity, prevalence of sexual problems, and associated helpseeking patterns in men and women aged 40-80 years in Korea: data from the Global Study of Sexual Attitudes and Behaviors (GSSAB). J Sex Med 2006;3:201-11.

17. Shechter A, Lowenstein L, Serefoglu EC, et al. Attitudes of Sexual Medicine Specialists Toward Premature Ejaculation Diagnosis and Therapy. Sex Med 2016;4:e209-16.

18. Shabsigh R. Diagnosing premature ejaculation: a review. J Sex Med 2006;3 Suppl 4:318-23.

19. Kandeel FR, Koussa VK, Swerdloff RS. Male sexual function and its disorders: physiology, pathophysiology, clinical investigation, and treatment. Endocr Rev 2001;22:342-88.

20. Rowland D, Perelman M, Althof S, et al. Self-reported premature ejaculation and aspects of sexual functioning and satisfaction. J Sex Med 2004;1:225-32.

21. Althof SE, Abdo CH, Dean J, et al. International Society for Sexual Medicine's guidelines for the diagnosis and treatment of premature ejaculation. J Sex Med 2010;7:2947-69.

22. Yuan YM, Xin ZC, Jiang H, et al. Sexual function of premature ejaculation patients assayed with Chinese Index of Premature Ejaculation. Asian J Androl 2004;6:121-6.

23. 2Arafa M, Shamloul R. Development and evaluation of the Arabic Index of Premature Ejaculation (AIPE). J Sex Med 2007;4:1750-6.

24. Dinsmore WW, Wyllie MG. PSD502 improves ejaculatory latency, control and sexual satisfaction when applied topically $5 \mathrm{~min}$ before intercourse in men with premature ejaculation: results of a phase III, multicentre, doubleblind, placebo-controlled study. BJU Int 2009;103:940-9.

25. McMahon CG, Althof SE, Kaufman JM, et al. Efficacy and safety of dapoxetine for the treatment of premature ejaculation: integrated analysis of results from five phase 3 trials. J Sex Med 2011;8:524-39.

26. Giuliano F, Patrick DL, Porst H, et al. Premature ejaculation: results from a five-country European observational study. Eur Urol 2008;53:1048-57.

27. Althof SE. Patient reported outcomes in the assessment of premature ejaculation. Transl Androl Urol 2016;5:470-4.

28. Seo DH, Jeh SU, Choi SM, et al. Diagnosis and Treatment of Premature Ejaculation by Urologists in South Korea. World J Mens Health 2016;34:217-23.

29. Association AP. Diagnostic and statistical manual of mental disorders (DSM-5®). American Psychiatric Pub; 2013.

30. Waldinger MD, Hengeveld MW, Zwinderman AH, et al. An empirical operationalization study of DSMIV diagnostic criteria for premature ejaculation. Int J Psychiatry Clin Pract 1998;2:287-93.

31. Serefoglu EC, Yaman O, Cayan S, et al. Prevalence of the complaint of ejaculating prematurely and the four premature ejaculation syndromes: results from the Turkish Society of Andrology Sexual Health Survey. J Sex Med 2011;8:540-8.

32. Gao J, Zhang X, Su P, et al. Prevalence and factors associated with the complaint of premature ejaculation and the four premature ejaculation syndromes: a large observational study in China. J Sex Med 2013;10:1874-81.

33. Godpodinoff ML. Premature ejaculation: clinical subgroups and etiology. J Sex Marital Ther 1989;15:130-4.

34. Serefoglu EC, Cimen HI, Atmaca AF, et al. The distribution of patients who seek treatment for the complaint of ejaculating prematurely according to the four premature ejaculation syndromes. J Sex Med 2010;7:810-5.

35. Parnham A, Serefoglu EC. Classification and definition of premature ejaculation. Transl Androl Urol 2016;5:416-23.

36. Waldinger MD, Schweitzer DH. The use of old and recent DSM definitions of premature ejaculation in observational studies: a contribution to the present 
debate for a new classification of PE in the DSM-V. J Sex Med 2008;5:1079-87.

37. Sotomayor $M$. The burden of premature ejaculation: the patient's perspective. J Sex Med 2005;2 Suppl 2:110-4.

38. Pryor J, Broderick G, Ho K, et al. Comparison of Estimated versus Measured Intravaginal Ejaculatory Latency Time (ielt) in Men with and without Premature Ejaculation (pe): 126. The Journal of Sexual Medicine 2006;3:54.

39. Patrick DL, Althof SE, Pryor JL, et al. Premature ejaculation: an observational study of men and their partners. J Sex Med 2005;2:358-67.

40. Rosen RC, McMahon CG, Niederberger C, et al. Correlates to the clinical diagnosis of premature ejaculation: results from a large observational study of men and their partners. J Urol 2007;177:1059-64; discussion 1064.

41. Cai L, Wen Y, Jiang M, et al. Premature ejaculation results from partners' mismatch: development and validation of index of intra-vaginal ejaculation latency time. Int J Impot Res 2016;28:101-5.

42. Althof SE, Rowland DL. Identifying constructs and criteria for the diagnosis of premature ejaculation: implication for making errors of classification. BJU Int 2008;102:708-12.

43. Bhat GS, Shastry A. New Tools to Measure Ejaculatory Latency-Arousal to Ejaculation Time Interval and Erection to Ejaculation Time Interval: A Pilot Study. Urology 2018;115:107-11.

44. Waldinger MD. Four measures of investigating ejaculatory performance. J Sex Med 2007;4:520.

45. Serefoglu EC, Cimen HI, Ozdemir AT, et al. Turkish validation of the premature ejaculation diagnostic tool and its association with intravaginal ejaculatory latency time. Int J Impot Res 2009;21:139-44.

46. Wyllie MG, Hellstrom WJ. The link between penile hypersensitivity and premature ejaculation. BJU Int 2011;107:452-7.

47. Martin CL, Waberski BH, Pop-Busui R, et al. Vibration perception threshold as a measure of distal symmetrical peripheral neuropathy in type 1 diabetes: results from the DCCT/EDIC study. Diabetes Care 2010;33:2635-41.

48. Xin ZC, Chung WS, Choi YD, et al. Penile sensitivity in patients with primary premature ejaculation. J Urol 1996;156:979-81.

49. Chen X, Wang FX, Hu C, et al. Penile sensory thresholds in subtypes of premature ejaculation: implications of comorbid erectile dysfunction. Asian J Androl 2018;20:330-5.
50. Vanden Broucke H, Everaert K, Peersman W, et al. Ejaculation latency times and their relationship to penile sensitivity in men with normal sexual function. J Urol 2007;177:237-40.

51. Rowland DL, Haensel SM, Blom JH, et al. Penile sensitivity in men with premature ejaculation and erectile dysfunction. J Sex Marital Ther 1993;19:189-97.

52. Salonia A, Sacca A, Briganti A, et al. Quantitative sensory testing of peripheral thresholds in patients with lifelong premature ejaculation: a case-controlled study. J Sex Med 2009;6:1755-62.

53. Paick JS, Jeong H, Park MS. Penile sensitivity in men with premature ejaculation. Int J Impot Res 1998;10:247-50.

54. Guo L, Liu Y, Wang X, et al. Significance of penile hypersensitivity in premature ejaculation. Sci Rep 2017;7:10441.

55. Xin ZC, Choi YD, Lee WH, et al. Penile vibratory threshold changes with various doses of SS-cream in patients with primary premature ejaculation. Yonsei Med J 2000;41:29-33.

56. Hill BJ, Janssen E, Kvam P, et al. The effect of condoms on penile vibrotactile sensitivity thresholds in young, heterosexual men. J Sex Med 2014;11:102-6.

57. Vardi Y, Gruenwald I, Sprecher E, et al. Normative values for female genital sensation. Urology 2000;56:1035-40.

58. Gao J, Zhang X, Su P, et al. The impact of intravaginal ejaculatory latency time and erectile function on anxiety and depression in the four types of premature ejaculation: a large cross-sectional study in a Chinese population. J Sex Med 2014;11:521-8.

59. Green SA, Rudie JD, Colich NL, et al. Overreactive brain responses to sensory stimuli in youth with autism spectrum disorders. J Am Acad Child Adolesc Psychiatry 2013;52:1158-72.

60. Xia JD, Zhou LH, Han YF, et al. A reassessment of penile sensory pathways and effects of prilocaine-lidocaine cream in primary premature ejaculation. Int J Impot Res 2014;26:186-90.

61. Xia JD, Jiang HS, Zhu LL, et al. Somatosensory evoked potentials assess the efficacy of circumcision for premature ejaculation. Int J Impot Res 2016;28:127-32.

62. Giuliano F, Rowland DL. Standard operating procedures for neurophysiologic assessment of male sexual dysfunction. J Sex Med 2013;10:1205-11.

63. Thomas AJ. Contemporary Management of Impotence and Infertility. JAMA 1989;261:1651-.

64. Xin ZC, Choi YD, Rha KH, et al. Somatosensory evoked potentials in patients with primary premature ejaculation. J 
Urol 1997;158:451-5.

65. Yilmaz U, Tatlisen A, Turan H, et al. The effects of fluoxetine on several neurophysiological variables in patients with premature ejaculation. J Urol 1999;161:107-11.

66. Zhou C, Jiang X, Xu Z, et al. Bulbocavernosus reflex to stimulation of prostatic urethra in patients with lifelong premature ejaculation. J Sex Med 2010;7:3750-7.

67. Fanciullacci F, Colpi GM, Beretta G, et al. Cortical evoked potentials in subjects with true premature ejaculation. Andrologia 1988;20:326-30.

68. Yang BB, Xia JD, Hong ZW, et al. No effect of abstinence time on nerve electrophysiological test in premature ejaculation patients. Asian J Androl 2018;20:391-5.

69. Tian L, Xin ZC, Xin H, et al. Effect of renewed SS-cream on spinal somatosensory evoked potential in rabbits. Asian J Androl 2004;6:15-8.

70. Xin ZC, Choi YD, Seong DH, et al. Sensory evoked potential and effect of SS-cream in premature ejaculation. Yonsei Med J 1995;36:397-401.

71. Perretti A, Catalano A, Mirone V, et al. Neurophysiologic evaluation of central-peripheral sensory and motor pudendal pathways in primary premature ejaculation. Urology 2003;61:623-8.

72. Xia JD, Han YF, Pan F, et al. Clinical characteristics and penile afferent neuronal function in patients with primary delayed ejaculation. Andrology 2013;1:787-92.

Cite this article as: Wei S, Wu C, Yu B, Ma M, Qin F, Yuan J. Advantages and limitations of current premature ejaculation assessment and diagnostic methods: a review. Transl Androl Urol 2020;9(2):743-757. doi: 10.21037/tau.2019.12.08
73. Jiang XZ, Zhou CK, Guo LH, et al. Role of bulbocavernosus reflex to stimulation of prostatic urethra in pathologic mechanism of primary premature ejaculation. Zhonghua Yi Xue Za Zhi 2009;89:3249-52.

74. Gao M, Yang X, Liu L, et al. Abnormal White Matter Microstructure in Lifelong Premature Ejaculation Patients Identified by Tract-Based Spatial Statistical Analysis. J Sex Med 2018;15:1272-9.

75. Xia JD, Han YF, Zhou LH, et al. Sympathetic skin response in patients with primary premature ejaculation. Int J Impot Res 2014;26:31-4.

76. Alwaal A, Breyer BN, Lue TF. Normal male sexual function: emphasis on orgasm and ejaculation. Fertil Steril 2015;104:1051-60.

77. Jannini EA, Ciocca G, Limoncin E, et al. Premature ejaculation: old story, new insights. Fertil Steril 2015;104:1061-73.

78. Herbert J. The role of the dorsal nerves of the penis in the sexual behaviour of the male rhesus monkey. Physiol Behav 1973;10:293-300.

79. Masters WH, Boston VEJ. Human sexual inadequacy. Psychoanalytic Quarterly 1973;42:139-45.

80. Rowland DL, Kallan K, Slob AK. Yohimbine, erectile capacity, and sexual response in men. Arch Sex Behav 1997;26:49-62. 